\title{
Internally Positive Representations and Stability Analysis of Linear Delay Systems with Multiple Time-Varying Delays
}

Francesco Conte, Vittorio De Iuliis, and Costanzo Manes

\begin{abstract}
This paper introduces the Internally Positive Representation of linear systems with multiple time-varying state delays. The technique, previously introduced for the undelayed case, aims at building a positive representation of systems whose dynamics is, in general, indefinite in sign. As a consequence, stability criteria for positive time-delay systems can be exploited to analyze the stability of the original system. As a result, an easy-to-check sufficient condition for the delay-independent stability is provided, that is compared with some well known conditions available in the literature.
\end{abstract}

\section{Introduction}

Positive linear systems have been extensively studied in the last decades due to their well known properties and applications $[6,18]$. More recently, several works on positive linear time-delay systems appeared in the literature, some of them providing insightful stability results $[1,12,15,17,16,23,19]$. To exploit the properties of positive systems also for not necessarily positive systems, an useful tool has recently been developed in the linear undelayed case: the Internally Positive Representation (IPR). The technique, introduced in the discrete-time framework in $[8,9,4]$ and in the continuous-time one in [2,3], aims at constructing internally positive representations of systems whose dynamics is indefinite in sign. The method presented in

F. Conte

Dipartimento di Ingegneria Navale, Elettrica, Elettronica e delle Telecomunicazioni, Università degli Studi di Genova, Via all'Opera Pia, 11A, I-16145 Genova (GE), Italy.

e-mail: fr.conte@unige.it

V. De Iuliis · C. Manes

Dipartimento di Ingegneria e Scienze dell'Informazione, e Matematica, Università degli Studi dell'Aquila, Via Vetoio, 67100 Coppito (AQ), Italy.

e-mail: vittorio.deiuliis@graduate.univaq.it, costanzo.manes@univaq.it 
[2], although very easy and straightforward, can produce in some cases an unstable positive system even if the original system is stable. Later works on the IPR focused on this issue, showing how to construct IPRs whose stability properties are equivalent to those of the original system [3].

As is typical in Systems and Control, one usually tries to extend to the more general case what is well known in the particular one: to this end, the main part of this paper focuses on the extension of the IPR construction method to linear continuous time-delay systems, in the general case of multiple time-varying delays. Then, a stability analysis follows, leading to the conclusion that only delay systems that are stable for any set of delays, constant or time-varying, can admit a stable IPR. As a result, an easy-to-check sufficient condition for the delay-independent stability of the original system is provided, whose efficacy with respect to other similar sufficient conditions available in the literature is tested by numerical examples.

This paper is organized as follows: in Section 2, the Internally Positive Representation for linear systems with multiple time-varying delays is introduced. Section 3 reports a discussion on the stability properties of IPRs and presents the new stability condition. In Section 4 the condition is compared with similar existing results, and in Section 5 an illustrative example is reported. Conclusions follow.

Notations. $\mathbb{R}_{+}$is the set of nonnegative real numbers. $\mathbb{C}^{-}$and $\mathbb{C}^{+}$are the open left-half and right-half complex planes, respectively. $\mathbb{R}_{+}^{n}$ is the nonnegative orthant of $\mathbb{R}^{n}$. $\mathbb{R}_{+}^{m \times n}$ is the cone of positive $m \times n$ matrices. $I_{n}$ is the $n \times n$ identity matrix. $\mathfrak{R}(z)$ and $\mathfrak{I}(z)$ are the real and imaginary parts of a complex number $z$, respectively. $\mathscr{C}\left([a, b], \mathbb{R}^{n}\right)$ denotes the Banach space of all continuous functions on $[a, b]$ with values in $\mathbb{R}^{n}$, endowed with the uniform convergence norm $\|\cdot\|_{\infty} . A \in \mathbb{R}^{n \times n}$ is said to be Metzler if all its off-diagonal elements are nonnegative. $d(A)$ denotes the diagonal matrix extracted from $A . \sigma(A)$ and $\alpha(A)$ denote the spectrum and the spectral abscissa of $A$, respectively. $A$ is said to be stable or Hurwitz if $\sigma(A) \subset \mathbb{C}^{-}$or, equivalently, if $\alpha(A)<0 . \mathscr{L}_{1}^{p}$ and $\mathscr{L}_{1,+}^{p}$ are the sets of locally integrable functions with values in $\mathbb{R}^{p}$ and $\mathbb{R}_{+}^{p}$, respectively. Finally, $\underline{m}=\{1,2, \ldots, m\}$ and $\underline{m}_{0}=\{0,1, \ldots, m\}$.

\section{Internally Positive Representation of Delay Systems}

\subsection{Internally positive delay systems}

Let $S=\left\{\left\{A_{k}\right\}_{0}^{m}, B, C, D\right\}_{n, p, q}$ denote a continuous-time delay system, with possibly time-varying delays, having the following form

$$
\begin{aligned}
& \dot{x}(t)=A_{0} x(t)+\sum_{k=1}^{m} A_{k} x\left(t-\delta_{k}(t)\right)+B u(t), \quad t \geq t_{0}, \\
& y(t)=C x(t)+D u(t), \\
& x(t)=\phi\left(t-t_{0}\right), \quad t \in\left[t_{0}-\delta, t_{0}\right],
\end{aligned}
$$


where $u(t) \in \mathbb{R}^{p}$ is the input, with $u \in \mathscr{L}_{1}^{p}, y(t) \in \mathbb{R}^{q}$ is the output, $x(t) \in \mathbb{R}^{n}$ is the system variable and $\phi \in \mathscr{C}\left([-\delta, 0], \mathbb{R}^{n}\right)$ is a pre-shape function (initial state). $\delta_{k}: \mathbb{R} \rightarrow \mathbb{R}_{+}$are time-delays, which are bounded continuous functions

$$
0 \leq \delta_{k}(t) \leq \delta, \quad \forall t \geq t_{0} .
$$

$B \in \mathbb{R}^{n \times p}, C \in \mathbb{R}^{q \times n}, D \in \mathbb{R}^{q \times p}$, and $A_{k} \in \mathbb{R}^{n \times n}$, for $k \in \underline{m}_{0}$. It is well known that the delay differential equation in (1) admits a unique solution satisfying a given initial condition $\phi$ (see e.g. [13]). Throughout the paper, the solution $x(t)$ and the corresponding output trajectory $y(t)$ associated to a system $S$ will be denoted as

$$
(x(t), y(t))=\Phi_{S}\left(t, t_{0}, \phi, u\right) .
$$

Following [14, 17], an internally positive linear delay system is defined as follows.

Definition 1 A delay system $S=\left\{\left\{A_{k}\right\}_{0}^{m}, B, C, D\right\}_{n, p, q}$ is said to be internally positive if

$$
\left\{\begin{array}{c}
\phi \in \mathscr{C}\left([-\delta, 0], \mathbb{R}_{+}^{n}\right) \\
u \in \mathscr{L}_{1,+}^{p}
\end{array}\right\} \Rightarrow\left\{\begin{array}{l}
x(t) \in \mathbb{R}_{+}^{n}, \\
y(t) \in \mathbb{R}_{+}^{q},
\end{array} \quad \forall t \geq t_{0}\right\} .
$$

Stated informally, $S$ is internally positive if nonnegative initial states and input functions produce nonnegative state and output trajectories. The following result gives necessary and sufficient conditions to fulfill Definition 1 (see [12, 23]).

Lemma 1 A delay system $S=\left\{\left\{A_{k}\right\}_{0}^{m}, B, C, D\right\}_{n, p, q}$ is internally positive if and only if $A_{0}$ is Metzler and $B, C, D$ and $A_{k}$, for $k \in \underline{m}$, are nonnegative.

\subsection{Positive representation of vectors and matrices}

Given a matrix (or vector) $M \in \mathbb{R}^{m \times n}$, the symbols $M^{+}, M^{-}$denote the componentwise positive and negative parts of $M$, while $|M|$ stands for its componentwise absolute value. It follows that $M=M^{+}-M^{-}$and $|M|=M^{+}+M^{-}$.

Let $\Delta_{n}=\left[I_{n}-I_{n}\right] \in \mathbb{R}^{n \times 2 n}$. The definitions reported below are taken from [9, 4].

Definition 2 A positive representation of a vector $x \in \mathbb{R}^{n}$ is any vector $\tilde{x} \in \mathbb{R}_{+}^{2 n}$ such that

$$
x=\Delta_{n} \tilde{x} .
$$

The min-positive representation of a vector $x \in \mathbb{R}^{n}$ is the positive vector $\pi(x) \in \mathbb{R}_{+}^{2 n}$ defined as

$$
\pi(x)=\left[\begin{array}{l}
x^{+} \\
x^{-}
\end{array}\right] .
$$

The min-positive representation of a matrix $M \in \mathbb{R}^{m \times n}$ is the positive matrix $\Pi(M) \in$ $\mathbb{R}_{+}^{2 m \times 2 n}$ defined as

$$
\Pi(M)=\left[\begin{array}{l}
M^{+} M^{-} \\
M^{-} M^{+}
\end{array}\right]
$$


while the min-Metzler representation of a matrix $A \in \mathbb{R}^{n \times n}$ is the Metzler matrix $\Gamma(A) \in \mathbb{R}^{2 n \times 2 n}$ defined as

$$
\Gamma(A)=\left[\begin{array}{cc}
d(A)+(A-d(A))^{+} & (A-d(A))^{-} \\
(A-d(A))^{-} & d(A)+(A-d(A))^{+}
\end{array}\right] .
$$

Of course, if $d(A) \in \mathbb{R}_{+}^{n \times n}$ then $\Gamma(A)=\Pi(A)$. Moreover, for any $x \in \mathbb{R}^{n}$ and matrices $M \in \mathbb{R}^{m \times n}, A \in \mathbb{R}^{n \times n}$ the following properties hold true:

(a) $x=\Delta_{n} \pi(x)$;

(b) $\Delta_{m} \Pi(M)=M \Delta_{n}$, so that $\Delta_{m} \Pi(M) \pi(x)=M x$;

(c) $\Delta_{n} \Gamma(A)=A \Delta_{n}$, so that $\Delta_{n} \Gamma(A) \pi(x)=A x$.

\subsection{Internally Positive Representations}

The concept of Internally Positive Representation (IPR) of an arbitrary system has been introduced in $[8,9,4]$, for discrete-time systems, and in [2,3] for continuoustime systems. The IPR construction presented in [2] can be extended to the case of time-varying delays systems by the following definition.

Definition 3 An Internally Positive Representation (IPR) of a delay system $S=$ $\left\{\left\{A_{k}\right\}_{0}^{m}, B, C, D\right\}_{n, p, q}$ is an internally positive system $\widetilde{S}=\left\{\left\{\widetilde{A}_{k}\right\}_{0}^{m}, \widetilde{B}, \widetilde{C}, \widetilde{D}\right\}_{\tilde{n}, \tilde{p}, \tilde{q}}$ together with four transformations $\left\{T_{X}^{f}, T_{X}^{b}, T_{U}, T_{Y}\right\}$,

$$
T_{X}^{f}: \mathbb{R}^{n} \mapsto \mathbb{R}_{+}^{\tilde{n}}, \quad T_{X}^{b}: \mathbb{R}_{+}^{\tilde{n}} \mapsto \mathbb{R}^{n}, \quad T_{U}: \mathbb{R}^{p} \mapsto \mathbb{R}_{+}^{\tilde{p}}, \quad T_{Y}: \mathbb{R}_{+}^{\tilde{q}} \mapsto \mathbb{R}^{q},
$$

such that $\forall t_{0} \in \mathbb{R}, \forall(\phi, u) \in \mathscr{C}\left([-\delta, 0], \mathbb{R}^{n}\right) \times \mathscr{L}_{1}^{p}$, the following implication holds:

$$
\left\{\begin{array}{c}
\tilde{\phi}(\tau)=T_{X}^{f}(\phi(\tau)), \forall \tau \in[-\delta, 0] \\
\tilde{u}(t)=T_{U}(u(t)), \forall t \geq t_{0}
\end{array}\right\} \Longrightarrow\left\{\begin{array}{l}
x(t)=T_{X}^{b}(\tilde{x}(t)), \quad \forall t \geq t_{0} \\
y(t)=T_{Y}(\tilde{y}(t)),
\end{array}\right\}
$$

where

$$
\begin{aligned}
& (x(t), y(t))=\Phi_{S}\left(t, t_{0}, \phi, u\right), \\
& (\tilde{x}(t), \tilde{y}(t))=\Phi_{\tilde{S}}\left(t, t_{0}, \tilde{\phi}, \tilde{u}\right) .
\end{aligned}
$$

$T_{X}^{f}$ and $T_{X}^{b}$ in (9) are the forward and backward state transformations of the IPR, respectively, while $T_{U}$ and $T_{Y}$ are the input and output transformations, respectively. The implication (10) means that if the (nonnegative) pre-shape function $\tilde{\phi}$ of the IPR is computed as the forward state transformation $T_{X}^{f}$ of the pre-shape function $\phi$ of the original system, and the (nonnegative) input $\tilde{u}$ to the IPR is computed as the input transformation $T_{U}$ of the input $u$ to the original system, then the state trajectory of the original system is given by the backward transformation $T_{X}^{b}$ of the (nonnegative) state $\tilde{x}$ of the IPR, and the output trajectory $y$ of the original system is 
given by the output transformation $T_{Y}$ of the (nonnegative) output $\tilde{y}$ of the IPR. For consistency, the backward map $T_{X}^{b}$ must be a left-inverse of the forward map $T_{X}^{f}$, i.e. $x=T_{X}^{b}\left(T_{X}^{f}(x)\right), \forall x \in \mathbb{R}^{n}$.

The following theorem provides a method for the IPR construction of arbitrary time-varying delays systems.

Theorem 2 Consider a delay system $S$ as in (1), with $S=\left\{\left\{A_{k}\right\}_{0}^{m}, B, C, D\right\}_{n, p, q}$. An internally positive system $\bar{S}=\left\{\left\{\bar{A}_{k}\right\}_{0}^{m}, \bar{B}, \bar{C}, \bar{D}\right\}_{2 n, 2 p, 2 q}$, with

$$
\bar{A}_{0}=\Gamma\left(A_{0}\right), \quad \bar{B}=\Pi(B), \quad \bar{C}=\Pi(C), \quad \bar{D}=\Pi(D), \quad \bar{A}_{k}=\Pi\left(A_{k}\right), \quad k \in \underline{m},
$$

together with the four transformations

$$
\begin{aligned}
\bar{x} & =T_{X}^{f}(x)=\pi(x), & x & =T_{X}^{b}(\bar{x})=\Delta_{n} \bar{x}, \\
\bar{u} & =T_{U}(u)=\pi(u), & y & =T_{Y}(\bar{y})=\Delta_{q} \bar{y},
\end{aligned}
$$

is an IPR of $S$.

Proof. First of all, since $\bar{A}_{0}$ is Metzler and $\bar{B}, \bar{C}, \bar{D}$, and $\bar{A}_{k}, k \in \underline{m}$, are all nonnegative, from Lemma 1 it follows that system $\bar{S}$ is internally positive. For any pre-shape function $\phi \in \mathscr{C}\left([-\delta, 0], \mathbb{R}^{n}\right)$, let $\bar{x}(t)$ and $\bar{y}(t)$ denote the state and output trajectories

$$
(\bar{x}(t), \bar{y}(t))=\Phi_{\bar{S}}\left(t, t_{0}, \bar{\phi}, \bar{u}\right)
$$

where $\bar{\phi}(\tau)=T_{X}^{f}(\phi(\tau))=\pi(\phi(\tau)), \forall \tau \in[-\delta, 0]$ and $\bar{u}(t)=T_{U}(u(t))=\pi(u(t))$, $\forall t \geq t_{0}$. Thus, (14) solves the system

$$
\begin{aligned}
& \dot{\bar{x}}(t)=\bar{A}_{0} \bar{x}(t)+\sum_{k=1}^{m} \bar{A}_{k} \bar{x}\left(t-\delta_{k}(t)\right)+\bar{B} \bar{u}(t), \quad t \geq t_{0}, \\
& \bar{y}(t)=\bar{C} \bar{x}(t)+\bar{D} \bar{u}(t), \\
& \bar{x}(t)=\bar{\phi}\left(t-t_{0}\right), \quad t \in\left[t_{0}-\delta, t_{0}\right] .
\end{aligned}
$$

Consider now the vectors

$$
\begin{aligned}
& z(t)=T_{X}^{b}(\bar{x}(t))=\Delta_{n} \bar{x}(t), \\
& v(t)=T_{Y}(\bar{y}(t))=\Delta_{q} \bar{y}(t) .
\end{aligned}
$$

The theorem is proved by showing that $x(t)=z(t)$ and $y(t)=v(t)$ for all $t \geq t_{0}$. Using properties (b) and (c), given in Section 2.2, and (16), it results that, for $t \geq t_{0}$,

$$
\begin{aligned}
\dot{z}(t)=\Delta_{n} \dot{\bar{x}}(t) & =\Delta_{n} \bar{A}_{0} \bar{x}(t)+\sum_{k=1}^{m} \Delta_{n} \bar{A}_{k} \bar{x}\left(t-\delta_{k}(t)\right)+\Delta_{n} \bar{B} \pi(u(t)) \\
& =A_{0} z(t)+\sum_{k=1}^{m} A_{k} z\left(t-\delta_{k}(t)\right)+B u(t) .
\end{aligned}
$$


and for $t \in\left[t_{0}-\delta, t_{0}\right]$

$$
z(t)=\Delta_{n} \bar{x}(t)=\Delta_{n} \bar{\phi}\left(t-t_{0}\right)=\Delta_{n} \pi\left(\phi\left(t-t_{0}\right)\right)=\phi\left(t-t_{0}\right),
$$

and

$$
v(t)=\Delta_{q} \bar{y}(t)=\Delta_{q} \bar{C} \bar{x}(t)+\Delta_{q} \bar{D} \pi(u(t))=C z(t)+D u(t), \quad t \geq t_{0} .
$$

Note that $(z(t), v(t))$ obey the same equations of (1), with the same initial condition. From the uniqueness of the solution we get $(z(t), v(t))=(x(t), y(t))$, and this concludes the proof.

Remark 1 If $A_{k}=0$ for all $k \in \underline{m}$ the IPR proposed in Theorem 2 coincides with the normal-form IPR proposed in [2] (Th. 4) for the delay-free case.

\section{Stability Analysis}

In this section we investigate the relationships between the stability of a delay system and of its IPR. A quite obvious consequence of the boundedness of the state transformations $T_{X}^{f}(\cdot)$ and $T_{X}^{b}(\cdot)$ in (12) is that if an IPR of a system is stable, then the original system is stable as well. As we will see, the converse is not always true.

Throughout this paper we will use a standard nomenclature about stability. The trivial solution $x(t) \equiv 0$ of a delay system of the type (1) is said to be stable if any solution $x(t)$ for all $t \geq t_{0}$ satisfies a bound of the type $\|x(t)\| \leq k\|\phi\|_{\infty}$, for some $k>0$. If in addition $\lim _{t \rightarrow \infty}\|x(t)\|=0$, the trivial solution is asymptotically stable. If there exist $k>0$ and $\eta>0$ such that $\|x(t)\| \leq k e^{-\eta t}\|\phi\|_{\infty}$, the trivial solution is said to be exponentially stable.

A delay system as in (1) is said to be stable if the trivial solution is asymptotically stable. It is worth recalling that the stability of a delay system of the type (1) depends on the nature of delays (see e.g. [7, 11]): one can have stability for a given set or for any set of constant delays, for commensurate constant delays, for time-varying delays, within a given bound or without a specific bound, fast or slowly varying, etc. For reasons that will soon be clear, in this paper we are mainly concerned with stability for any set of constant or time-varying delays without a specific bound (delay-independent stability).

\subsection{Stable IPRs of delay-free systems}

For the case of delay-free systems $\left(A_{k}=0, k \in \underline{m}\right)$ in [2] it has been shown that the IPR construction method there presented when applied to stable systems in some cases may produce unstable IPRs. Indeed, the spectrum of $\bar{A}_{0}=\Gamma\left(A_{0}\right)$ properly 
contains the spectrum of $A_{0}$, and the additional eigenvalues can be unstable. However, a change of coordinates on the original system can generally affect the stability of the IPR, and this fact can be exploited to obtain stable IPRs. In [2] it has been proved that such a change of coordinates exists if $\sigma\left(A_{0}\right)$ belongs to the sector of $\mathbb{C}^{-}$ characterized by $\Re(z)+|\mathfrak{I}(z)|<0$. In [3], the IPR construction method of [2] has been suitably extended so that stable IPRs can be constructed for any stable system, without any limitation on the location of the eigenvalues of $A_{0}$ within $\mathbb{C}^{-}$.

\subsection{Stability of positive delay-systems}

The IPR produced by the method in Theorem 2 is by construction a linear positive delay system. For this reason we recall below the stability conditions for such a class of systems. Consider a system of the type (1) which is internally positive (i.e., $A_{0}$ is Metzler and $A_{k}, k \in \underline{m}$, are nonnegative, Lemma 1). In [12] it has been proved that, when the delays $\delta_{k}$ are constant, a necessary and sufficient stability condition is that there exist $p$ and $r$ in $\mathbb{R}^{n}$ such that

$$
\left(\sum_{k=0}^{m} A_{k}\right)^{T} p+r=0 \quad p>0, \quad r>0 .
$$

Note that, being $\sum_{k=0}^{m} A_{k}$ a Metzler matrix, condition (21) is equivalent to $\sum_{k=0}^{m} A_{k}$ Hurwitz, i.e.

$$
\alpha\left(\sum_{k=0}^{m} A_{k}\right)<0
$$

Another interesting equivalent condition (see [6]), that does not require the explicit computation of eigenvalues (condition (22)) or solving a linear problem (condition (21)) is that all the leading principal minors of the matrix

$$
M=-\sum_{k=0}^{m} A_{k}
$$

are positive, i.e. $M_{i}>0$ for $i=1, \ldots, n$, where $M_{i}$ is the determinant of the matrix obtained removing the last $n-i$ rows and columns from $M$. Note that all these equivalent conditions do not depend on the size of the delays. In [1] and in [17] it has been proved that (22) is necessary and sufficient for stability even in the case of time-varying delays $\delta_{k}(t)$, without limitation on the size of the delays and of their derivatives. Ngoc in [23] proved a similar condition also for the case of distributed delays.

Remark 2 It should be remarked that condition (22) is necessary and sufficient for the delay-independent stability of a positive delay-system, while it is only necessary for the stability of general (not necessarily positive) systems, being required for the stability of the associated delay-free system. 
To summarize, we have the following:

Proposition 3 If a system $S$ as in (1), with $A_{0}$ Metzler and $B, C, D, A_{k}$, for $k \in \underline{m}$, nonnegative, is stable for a given set of constant delays $\delta_{k}$, then it is also delayindependent stable, i.e. stable for any arbitrary set of constant or time-varying delays.

Liu and Lam [16] showed that if a positive delay system is stable for all continuous and bounded delays, then the trivial solution is exponentially stable for all continuous and bounded delays. On the other hand, if the delays are continuous but unbounded, the trivial solution may be asymptotically stable but not exponentially stable.

\subsection{Stable IPRs of delay systems}

Consider the equations (15) of the IPR given in Theorem 2. We have the following:

Theorem 4 If a delay system $S$ as in (1) admits a stable IPR, then necessarily $S$ is delay-independent stable.

Proof. As discussed in the previous paragraph, since the IPR is a positive delay system, a necessary and sufficient condition for its stability is that the Metzler matrix $\sum_{k=0}^{m} \bar{A}_{k}$ is Hurwitz, and this in turn implies that the IPR is delay-independent stable. The boundedness of the state transformations $T_{X}^{f}(\cdot)$ and $T_{X}^{b}(\cdot)$ defined in (12) trivially implies the delay-independent stability of the original system.

Stated in another way, Theorem 4 claims that only delay systems that are delayindependent stable admit stable Internally Positive Representations.

Theorem 4 suggests the following sufficient condition of delay-independent stability for not necessarily positive delay systems.

Theorem 5 Consider a delay system $S$ as in (1). If

$$
\alpha\left(\Gamma\left(A_{0}\right)+\sum_{k=1}^{m} \Pi\left(A_{k}\right)\right)<0
$$

then $S$ is delay-independent stable.

Proof. Note first that the Metzler matrix in (23) coincides with $\sum_{k=0}^{m} \bar{A}_{k}$, where $\bar{A}_{k}$ are the matrices of the IPR of Theorem 2. Thus, if condition (23) is satisfied, then the IPR of $S$ is stable, and thanks to Theorem 4 the original system $S$ is delayindependent stable.

Remark 3 As pointed out in Section 3.2, checking condition (23) does not require the explicit computation of the eigenvalues of the Metzler matrix $\Gamma\left(A_{0}\right)+$ $\sum_{k=1}^{m} \Pi\left(A_{k}\right)$. Indeed, an easy equivalent condition only requires to check that all the leading principal minors of $M=-\left(\Gamma\left(A_{0}\right)+\sum_{k=1}^{m} \Pi\left(A_{k}\right)\right)$ are positive. 


\section{Comparison with similar conditions of delay-independent stability}

Many stability conditions exist for delay systems with multiple delays, based on different techniques: frequency sweeping [5], spectral analysis [20], Linear Matrix Inequalities [10,7] and others (see [11]). These results refer to different cases such as commensurate or incommensurate delays, constant or time-varying delays, slowly or fast varying delays. Many stability tests rely on numerical computations and some have a not negligible computational complexity (particularly the necessary and sufficient ones). Coming to delay-independent stability, in [21] and [22], for the case of single and constant delay, the following sufficient condition for delay-independent stability has been given

$$
\mu_{p}\left(A_{0}\right)+\left\|A_{1}\right\|_{p}<0
$$

where $\mu_{p}(A)$ is the logarithmic norm (or measure) of matrix $A$ induced by the operator norm $\|A\|_{p}$, defined as:

$$
\mu_{p}(A)=\lim _{\varepsilon \rightarrow 0} \frac{\|I+\varepsilon A\|_{p}-1}{\varepsilon} .
$$

The expression of $\mu_{p}(\cdot)$ can easily be computed for $p=1,2, \infty$ :

$$
\begin{aligned}
& \mu_{1}(A)=\max _{j=1 \ldots n}\left(a_{i i}+\sum_{i=1, i \neq j}^{n}\left|a_{i j}\right|\right), \\
& \mu_{2}(A)=\frac{1}{2} \lambda_{\max }\left(A^{T}+A\right), \\
& \mu_{\infty}(A)=\max _{i=1 \ldots n}\left(a_{i i}+\sum_{j=1, j \neq i}^{n}\left|a_{i j}\right|\right) .
\end{aligned}
$$

The extended condition:

$$
\mu_{p}\left(A_{0}\right)+\sum_{k=1}^{m}\left\|A_{k}\right\|_{p}<0
$$

has been shown [26] to be sufficient for the stability of systems with multiple commensurate delays, although only for the case of $p=2$. In [25] and [24] the same condition has been proven sufficient, for any $p$, also in the case of non commensurate and time-varying delays of any size, and therefore is a sufficient condition of delay-independent stability of the system.

As a matter of fact, it is rather easy to find delay-independent stable systems which satisfy condition (23) given in Theorem 5 and do not satisfy condition (25): an example is reported in Section 5. Further investigations are needed to compare the conservativeness of the new condition with respect to the classical one. 


\section{Example}

Consider the problem of verifying the delay-independent asymptotic stability of a system $S=\left\{\left\{A_{0}, A_{1}, A_{2}\right\}, B, C, D\right\}_{3, p, q}$ with:

$A_{0}=\left[\begin{array}{rrr}-25 & -5 & -14 \\ 0 & -19 & 0.1 \\ 0.7 & 1.2 & -16\end{array}\right], \quad A_{1}=\left[\begin{array}{rrr}-1.5 & -0.4 & 0 \\ 0.5 & -2.9 & 1 \\ -1.5 & 0.5 & -3.4\end{array}\right], \quad A_{2}=\left[\begin{array}{ccc}-7 & 2 & 6.8 \\ 1.8 & -1.6 & -2.1 \\ 0.5 & 1.6 & -3.3\end{array}\right]$

Since $S$ is not an internally positive system, (22) is only a necessary condition for its delay-independent stability (see Remark 2). We have that:

$$
\alpha\left(\sum_{k=0}^{m} A_{k}\right)=-23.131<0
$$

and therefore condition (22) is satisfied. Hence we can check the proposed sufficient condition (23), verifying that all the leading principal minors of the matrix

$$
M=-\left(\Gamma\left(A_{0}\right)+\Pi\left(A_{1}\right)+\Pi\left(A_{2}\right)\right)
$$

are positive (Remark 3). We get:

$$
\begin{aligned}
& M_{1}=25, \quad M_{2}=470.4, \quad M_{3}=7.2 \cdot 10^{3}, \quad M_{4}=1.4 \cdot 10^{5}, \\
& M_{5}=2.3 \cdot 10^{6}, \quad M_{6}=\operatorname{det}(M)=1.5 \cdot 10^{7}
\end{aligned}
$$

and this is sufficient to conclude that the system is delay-independent stable. Actually, the exact value of condition (23) is:

$$
\alpha\left(\Gamma\left(A_{0}\right)+\Pi\left(A_{1}\right)+\Pi\left(A_{2}\right)\right)=-2.436
$$

It is not possible to achieve the same conclusion on the stability of the system applying the classical sufficient condition (25), since:

$$
\begin{array}{r}
\mu_{1}\left(A_{0}\right)+\left\|A_{1}\right\|_{1}+\left\|A_{2}\right\|_{1}=14.700>0, \\
\mu_{2}\left(A_{0}\right)+\left\|A_{1}\right\|_{2}+\left\|A_{2}\right\|_{2}=2.896>0, \\
\mu_{\infty}\left(A_{0}\right)+\left\|A_{1}\right\|_{\infty}+\left\|A_{2}\right\|_{\infty}=15.200>0,
\end{array}
$$

and therefore the condition is not satisfied at least for $p=1,2, \infty$.

To sum up, for the system in this example the criterion (25) fails to assess the stability, which has been proved using the proposed condition (23).

Figure 1 depicts some examples of time evolution of $\log (\|x(t)\|)$ obtained with $u(t)=0$ for $t \in[0,200]$ and different constant values of the two delays. In all cases, the plotted quantity decreases linearly, thus confirming the asymptotic stability, which in the case of constant delays is also exponential. 


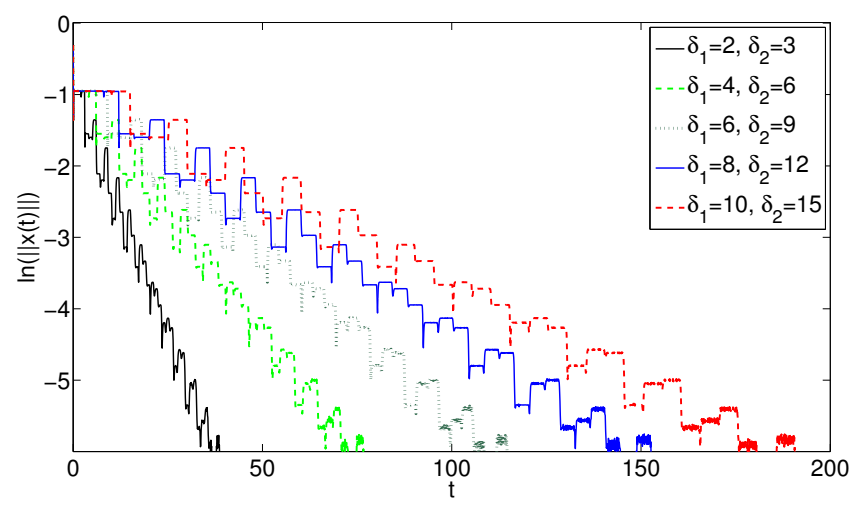

Fig. 1 Plot of $\log (\|\mathbf{x}(\mathbf{t})\|)$ with different constant delays values.

\section{Conclusions and future work}

In this paper the Internally Positive Representation of linear delay systems with multiple delays, possibly time-varying, has been introduced, and its consequences on the study of the stability of the original system have been investigated, leading to an easy-to-check sufficient condition whose efficacy with respect to the delayindependent stability tests provided in $[21,24,25]$ has been tested by means of numerical examples. Future work will be devoted to further stability analysis and to the extension of the IPR technique to other classes of delay systems.

\section{Acknowledgments}

We would like to thank Alfredo Germani and Filippo Cacace for their encouragement and helpful suggestions in doing this work.

\section{References}

1. Ait Rami, M.: Positive Systems: Proceedings of the third Multidisciplinary International Symposium on Positive Systems: Theory and Applications (POSTA 2009) Valencia, Spain, September 2-4, 2009, pp. 205-215. Springer Berlin Heidelberg, Berlin, Heidelberg (2009)

2. Cacace, F., Farina, L., Germani, A., Manes, C.: Internally positive representation of a class of continuous time systems. IEEE Transactions on Automatic Control 57(12), 3158-3163 (2012)

3. Cacace, F., Germani, A., Manes, C.: Stable internally positive representations of continuous time systems. IEEE Transactions on Automatic Control 59(4), 1048-1053 (2014)

4. Cacace, F., Germani, A., Manes, C., Setola, R.: A new approach to the internally positive representation of linear MIMO systems. IEEE Transactions on Automatic Control 57(1), 119-134 (2012)

5. Chen, J., Latchman, H.A.: Frequency sweeping tests for stability independent of delay. IEEE Transactions on Automatic Control 40(9), 1640-1645 (1995)

6. Farina, L., Rinaldi, S.: Positive linear systems: theory and applications, vol. 50. John Wiley \& Sons (2011) 
7. Fridman, E.: Introduction to Time-Delay Systems. Birkhuser Basel (2014)

8. Germani, A., Manes, C., Palumbo, P.: State space representation of a class of MIMO systems via positive systems. In: Decision and Control, 2007 46th IEEE Conference on, pp. 476-481. IEEE (2007)

9. Germani, A., Manes, C., Palumbo, P.: Representation of a class of MIMO systems via internally positive realization. European Journal of Control 16(3), 291-304 (2010)

10. Gu, K., Kharitonov, V.L., Chen, J.: Stability of Time-Delay Systems. Birkhuser Basel (2003)

11. Gu, K., Niculescu, S.I.: Survey on recent results in the stability and control of time-delay systems. Journal of Dynamic Systems, Measurement, and Control 125(2), 158-165 (2003)

12. Haddad, W.M., Chellaboina, V.: Stability theory for nonnegative and compartmental dynamical systems with time delay. Systems \& Control Letters 51(5), 355 - 361 (2004)

13. Hale, J., Lunel, S.M.V.: Introduction to Functional Differential Equations. New York: Springer-Verlag (1993)

14. Kaczorek, T.: Positive 1D and 2D Systems. London UK: Springer-Verlag (2001)

15. Kaczorek, T.: Stability of positive continuous-time linear systems with delays. In: Control Conference (ECC), 2009 European, pp. 1610-1613. IEEE (2009)

16. Liu, X., Lam, J.: Relationships between asymptotic stability and exponential stability of positive delay systems. Int. J. General Systems 42(2), 224-238 (2013)

17. Liu, X., Yu, W., Wang, L.: Stability analysis for continuous-time positive systems with timevarying delays. IEEE Transactions on Automatic Control 55(4), 1024-1028 (2010)

18. Luenberger, D.: Introduction to dynamic systems: theory, models, and applications. Wiley (1979)

19. Mazenc, F., Malisoff, M.: Stability analysis for time-varying systems with delay using linear Lyapunov functionals and a positive systems approach. IEEE Transactions on Automatic Control 61(3), 771-776 (2016)

20. Michiels, W., Niculescu, S.I.: Stability, Control, and Computation for Time-Delay Systems: An Eigenvalue-Based Approach, vol. 27. Siam (2014)

21. Mori, T., Fukuma, N., Kuwahara, M.: Simple stability criteria for single and composite linear systems with time delays. International Journal of Control 34(6), 1175-1184 (1981)

22. Mori, T., Fukuma, N., Kuwahara, M.: On an estimate of the decay rate for stable linear delay systems. International Journal of Control 36(1), 95-97 (1982)

23. Ngoc, P.H.A.: Stability of positive differential systems with delay. IEEE Transactions on Automatic Control 58(1), 203-209 (2013)

24. Schoen, G.M.: Stability and stabilization of time-delay systems. Ph.D. thesis, Swiss Federal Institute of Technology, Zurich, Switzerland (1995). Http://e-collection.library.ethz.ch

25. Schoen, G.M., Geering, H.P.: On stability of time delay systems. In: Proc. of the 31 st Annual Allerton Conf. on Comm., Control and Comp., pp. 1058-1060. Monticello, Il (1993)

26. Wang, S.S., Lee, C.H., Hung, T.H.: New stability anlysis of system with multiple time delays. In: American Control Conference (ACC 1991), pp. 1703 - 1704 (1991) 